\title{
O CONSUMO DE DROGAS ENTRE OS ESTUDANTES ADOLESCENTES
}

\author{
DRUG ABUSE AMONG FROM THE SEVENTH AND EIGHTH SERIES STUDENTS IN \\ THE CITY OF ALFENAS
}

Denis da Silva Moreira ${ }^{1}$

RESUMO: Este trabalho de pesquisa aborda os aspectos relacionados ao consumo de drogas entre os estudantes de $7^{\circ}$ e $8^{\circ}$ séries, na faixa etária de 12 a 15 anos, das escolas públicas e particulares da cidade de Alfenas. no ano de 1993. As drogas mais oferecidas e mais usadas foram os solventes voláteis ou inalantes, seguidos da maconha. O local onde a oferta é mais freqüente são os iugares púlulicos / ginásios de show, bares, casa de jogos, parques e outros 1. Constatou-se que 5.5\% dos entrevistados já fizeram uso de drogas, sendo motivados pela curiosidade e influência dos amigos. Praticamente łodos os estudantes já receberam, através da familia e/ou escola, orientação sobre drogas. A escola é a instituição que possui a maior clientela de risco às drogas, ou seja, crianças, adolescentes e jovens e por ser uma das responsáveis na educação e em passar cultura é a que possui maiores condições de executar um programa de prevenção às drogas.

UNITERMOS: Drogas - Estudarites - Escola

\section{INTRODUÇÃO}

A droga é tão antiga como a própria humanidade. Callo (1984) descreve que, no Oriente, o homem extraía o ópio da cápsula da papoula (Papaver somniferum). Heródoto conta que os Citas se embriagavam com os vapores das sementes do cânhamo que eram lançadas sobre pedras aquecidas, o que demonstrava na antigüidade já existir o vício da maconha. A planta, sagrada para os Indus, também era tida como divina por certas tribos africanas, donde vieram as sementes para o Brasil, nas tangas do escravos. Desde então surgiu o uso das drogas, por várias razões ligado às culturas dos povos: rituais religiosos, comemorações e busca do prazer.

Para Charbonneau (1983), todos precisam enfrentar uma nova luta que as gerações anteriores não conheceram: a luta contra a droga. Há mais ou menos $2 \mathrm{O}$ anos que a droga se tornou um mal generalizado que se impõe em todos os países, em todos os meios, em todas as classes sociais. "Já não é mais o jovem que procura o tóxico, tentando aliviar a carga de suas angústias existenciais; é o tóxico que chega ao jovem, angustiado ou não, e lhe é oferecido como um produto, um simples produto de consumo, que enriquece governos, grupos

\footnotetext{
' Enfermeiro do Departamento de Enfermagem e (Obstetricia da Fscola de Farmácia e (Odontologia de Alfenas. Enfermeiro do Hospital Universitário Alzira V'clano.
} 
poderosos, policiais de todos os niveis e os traficantes profissionais, deixando um saldo trágico de paranóicos $€$ mortos-vivos entre os consumidores, as únicas vítimas reais dessa rendosa multinacional". Assim tornou-se impossivel não se perceber a questão das drogas. É, antes de tudo, um problema social.

Segundo Murad (1972), é significante o dado de que vários indivíduos que abusam das drogas têm sua primeira experiência na época da puberdade. É a época em que há o desaparecimento dos laços familiares, a diminuição da autoridade paterna, a maturação sexual e o aumento da responsabilidade pessoal. Cercado pela ansiedade, frustração, medo do fracasso, além de conflitos internos e dúvidas atrozes, o adolescente talvez julgue que as "drogas" possam ajudá-lo a melhorar as suas amizades, facilitar a sua conversação, aumentar sua percepção, produzir alívio e fuga.

A droga deixou de ser um fenômeno de marginalização para passar a ser um fenômeno de cultura e, por isso, a juventude é a primeira a ser atingida. O seu primeiro contato com a comunidade humana fora da família é a escola. É nela que adquire a sua cultura, através dos educadores, dos colegas, da comunidade escolar no seu sentido mais amplo. Por causa disso estamos frente a uma situação da qual devemos nos tornar muito conscientes, Charbonneau (1987). As drogas começaram a circular pelas escolas do país por volta da década de 60 , mas a situação hoje é dramática. Nunca foi tão fácil comprar drogas nos colégios, nunca foi tão ampla a rede de traficantes entre os estudantes e nunca foi tão difícil controlar esse tipo de comércio, afirma o delegado Abilio Pereira (VEJA, 1991).

Para Vizzolto (1987), a escola é a instituição que retém a clientela de maior risco frente às drogas, ou seja, crianças, adolescentes e jovens. Portanto, para Charbonneau (1983), "é na escola que se deve estabelecer uma política preventiva que faça com que o jovem vulnerável seja capaz de fazer uma opção consciente".

Não se trata, pois, de um discurso proibitivo, nem permissivo, mas informativo e sério, de forma objetiva, não com a finalidade de criar pânico, nem identificar num discurso comum todas as drogas, mas, conforme Murad (1985), a campanha deve ser educativa e em bases fundamentadas para um bom esclarecimento. As informações podem ter um cunho popular, mas os fundamentos devem ser estritamente científicos.

Farias (1992) comenta que nas últimas décadas se tem observado a preocupação de grande número de países em realizar estudos sobre o consumo de drogas na população, objetivando implementar políticas públicas sobre a questão.

Assim, a presente pesquisa justifica-se por não existirem estudos sobre o consumo de drogas pelos estudantes de $1^{\circ}$ Grau na cidade de Alfenas. Esta informação é necessária pelo fato de Alfenas ser uma cidade que possui um número significativo de estudantes. 


\section{OBJETIVOS}

Esta pesquisa tem como objetivos:

- estudar o consumo de drogas entre os estudantes de $7^{\mathrm{a}}$ e $8^{\mathrm{a}}$ séries, matriculados regularmente nas escolas da cidade de Alfenas,

- identificar os tipos de drogas mais utilizadas pelos estudantes da referida pesquisa,

- verificar se estes estudantes recebem alguma orientação e qual é a fonte dessa orientação,

- comparar os resultados obtidos com outros trabalhos similares realizados em nível nacional e internacional.

\section{MATERIAL E MÉTODO}

\section{MATERIAL}

\section{POPULAÇÃO E AMOSTRA}

A população compreende 1044 estudantes matriculados nas $7^{\mathrm{a}}$. e $8^{\mathrm{a}}$. séries das seis escolas de $1^{\circ} \mathrm{Grau}$ da cidade de Alfenas, com idade entre 12 e 34 anos.

Utilizou-se uma amostragem não probabilistica, tipo intencional, que se constituiu de 774 estudantes de $7^{\mathrm{a}}$. e $8^{\mathrm{a}}$. séries, na faixa etária de doze (12) a quinze (15) anos. A definição da faixa etária baseou-se em várias bibliografias que indicavam significativa a utilização de drogas na adolescência Sanches (1982), afirmando ser grande o consumo de tóxicos na escola e seus índices aumentarem dia a dia.

\section{MÉTODO}

\section{INSTRUMENTO DE MEDIDA}

O questionário utilizado foi constituído por questões estruturadas. $\mathrm{Na}$ construção elaboração deste instrumento de medida, "a priori" montou-se uma entrevista não estruturada, não dirigida, com a participação dez(10) estudantes de $7^{\mathrm{a}}$. e $8^{\mathrm{a}}$. séries, visando conhecer 0 universo a ser pesquisado. Posteriormente, foram realizadas quatro (4) entrevistas (exploratórias) com questões semi-estruturadas, verificando-se se havia compreensão e clareza na aplicação do instrumento elaborado.

\section{COLETA DE DADOS}

A coleta de dados foi realizada em seis (6) escolas da cidade de Alfenas entre os estudantes de $7^{\circ}$ e $8^{\circ}$ séries no seu período de aula, tanto diurno quanto noturno, nos dias 22, 23 e 24 de março de 1993. 
Os dados foram colhidos pelo autor da pesquisa auxiliado por uma professora. Após uma breve explicação sobre 0 preenchimento dos questionários e informar que estes seriam sigilosos, cada estudante em média, usou 10 minutos para responder ao questionário.

Durante a aplicação dos questionários, não houve o surgimento de dúvidas em relação à compreensão do instrumento de medida.

A tabulação foi realizada manualmente. Os dados apresentados de acordo com os resultados foram descritos e analisados estatisticamente ao nivel de $5 \%$ de probabilidade através do teste $X^{2}$ (Qui-Quadrado).

\section{RESULTADOS E DISCUSSÃO}

Após uma análise detalhada dos 774 questionários, chegou-se aos seguintes resultados.

Entre os estudantes das escolas públicas entrevistados, cerca de $18 \%$ responderam que já the ofereceram drogas, mas $82 \%$ afirmaram que não. Nas escolas particulares, $22 \%$ responderam que já lhe ofereceram drogas e $78 \%$ dos respondentes disseram que nunca the ofertaram algum tipo de drogas(TAB.1).

Analisada estatisticamente, observa-se que a oferta de drogas é a mesma para os estudantes das escolas públicas como para os das escolas particulares.

TABELA 1 - PROPORÇÃO DE OFERTA DE DROGAS ENTRE OS ESTUDANTES DE $7^{\mathrm{a}}$ E $8^{\mathrm{a}}$ SÉRIES DO $1^{\circ}$ GRAU, COM IDADE ENTRE 12 A 15 ANOS, ALFENAS - MG. - 1993.

\begin{tabular}{c|c|c|c|c|}
\hline \multirow{2}{*}{ OFERECERAM } & \multicolumn{4}{|c}{ ESCOLAS } \\
\cline { 2 - 5 } & \multicolumn{2}{|c|}{ PÚBL.ICAS } & \multicolumn{2}{c}{ PARTICULARES } \\
\cline { 2 - 5 } & F & $\%$ & F & $\%$ \\
\hline SIM & 101 & 18,0 & 047 & 22,0 \\
NÃO & 459 & 82,0 & 167 & 78,0 \\
\hline TOTAL & 560 & 100,0 & $\mathbf{2 1 4}$ & 100,0 \\
\hline
\end{tabular}

Questionando-se sobre quals drogas foram ofertadas aos estudantes, obteve-se que os solventes voláteis ou inalantes vêm em primeiro lugar com $67,6 \%$ das ofertas, a maconha com $28 \%$, a cocaina em terceiro com $3,6 \%$ e duas substâncias curiosas como a semente de guaraná $0,4 \%$ e uma substância denominada de censo, $0,4 \%$, não sendo possivel identificar esta droga.

A TABELA 2 evidencia que o local de oferta de drogas não é significativamente diferente nas escolas públicas e particulares. 
TABELA 2 - DISTRIBUIÇÃO DOS LOCAIS ONDE FORAM OFERTADAS AS DROGAS AOS ESTUDANTES DE $7^{\mathrm{a}}$ E $8^{\mathrm{a}}$ SÉRIES, COM IDADE ENTRE 12 A 15 ANOS. ALFENAS - MG - 1993.

\begin{tabular}{|c|c|c|c|c|}
\hline \multirow{3}{*}{$\begin{array}{l}\text { LOCAIS DA OFERTA } \\
\text { DE DROGAS }\end{array}$} & \multicolumn{4}{|c|}{ ESCOLAS } \\
\hline & \multicolumn{2}{|c|}{ PÚBLICAS } & \multicolumn{2}{|c|}{ PA.RTICULARES } \\
\hline & $\mathbf{F}$ & $\%$ & F & $\%$ \\
\hline CASA DE AMIGO & 16 & 12,8 & 05 & 10,0 \\
\hline ESCOLA & 06 & 04,8 & 01 & 02,0 \\
\hline FESTA & 30 & 24,0 & 11 & 22,0 \\
\hline LUGAR PÚBLICO & 73 & 58,4 & 33 & 66,0 \\
\hline TOTAL & 125 & 100,0 & 50 & 100,0 \\
\hline
\end{tabular}

Segundo os resultados da presente pesquisa, a escola foi o último local onde os estudantes receberam ofertas de drogas. Constatou-se que a maior oferta de drogas foi nos lugares públicos (ginásios de show, bares, casa de jogos, parques etc). Para Sanchez (1982), tal comportamento justifica-se pelo receio de o aluno ser pego em flagrante por algum vigilante ou professor. Isso implicaria no conhecimento dos pais, eventualmente no da comunidade e nas conseqüências que daí pudessem advir. Uma outra suposição seria a de que fora da escola o aluno se sente mais estimulado ao uso da droga e que esses ambientes são propiciadores de uma atmosfera mais adequada e predisponente ao consumo que $o$ ambiente escolar.

A TABELA 3 mostra que $5 \%$ dos estudantes da escolas públicas responderam que já usaram algum tipo de droga. No caso das escolas particulares, $7 \%$ dos entrevistados disseram que já usaram algum tipo de droga. Comparando a proporção de estudantes que usaram drogas, verificou-se que é a mesma tanto nas escolas públicas como nas particulares.

TABELA 3 - PERCENTUAL DE ESCOLARES DE $7^{\mathrm{a}}$ E $8^{\mathrm{a}}$ SÉRIES, NA FAIXA ETÁRIA DE 12 A 15 ANOS, QUE JÁ USARAM DROGAS, ALFENAS - MG. - 1993.

\begin{tabular}{|c|c|c|c|c|}
\hline \multirow{3}{*}{ USO DEDROGAS } & \multicolumn{4}{|c|}{ ESCOLAS } \\
\hline & \multicolumn{2}{|c|}{ PÚBLICAS } & \multicolumn{2}{|c|}{ PARTICULARES } \\
\hline & $\mathbf{F}$ & $\%$ & $\mathbf{F}$ & $\%$ \\
\hline SIM & 028 & 05,0 & 015 & 07,0 \\
\hline NÃO & 532 & 95,0 & 199 & 93,0 \\
\hline TOTAL & 560 & 100,0 & 214 & 100,0 \\
\hline
\end{tabular}


Farias (1992) cita um estudo voltado para o consumo de drogas por estudantes realizado por Murad em 1979 na cidade de Belo Horizonte e em mais nove cidades do Estado de Minas Gerais, o qual se restringiu a indagar aos estudantes se eles usam ou já haviam usado drogas - o que, no entender de Morgado (apud Farias,1992), limitou o alcance do estudo. Todavia a informação de "usa ou já usou drogas", por um grupo populacional, é importante porque permite calcular as taxas de prevalência de drogas vigentes na época do estudo. A amostra desse estudo constou de 1664 estudantes secundaristas. Concluiu-se que, aproximadamente, $16 \%$ dos alunos usavam ou já haviam usado drogas e que $84 \%$ nunca usaram.

Outro estudo importante no que diz respeito ao consumo de drogas por estudantes é o relatório de Costa et al.(apud Farias 1992) que realizaram um inquérito epidemiológico entre 433.360 escolares da região metropolitana de São Paulo, verificando-se que no global as taxas de consumo são baixas. Embora as percentagens de uso de drogas ilícitas constantes nestes estudos não sejam elevadas, elas são importantes, especialmente porque um número significativo de escolares consome ou já consumiu, de forma habitual, cocaina, alucinógenos e narcóticos. Quanto a essa constatação, Morgado (apud Farias,1993) comenta que esses niveis de consumo estão muito aquém dos observados entre os estudantes da mesma faixa etária dos Estados Unidos, Canadá e paises europeus.

Segundo o National Institute On Drug Abuse (apud Wordaki,1990), 70 milhões de americanos, ou $37 \%$ do total da população americana com idade de 12 anos ou mais, já usaram maconha, cocaina ou outra droga ilícita em algum momento de suas vidas. Vinte e três milhões, ou $12 \%$, eram usuários correntes, isto é, haviam usado uma droga ilegal dentro dos últimos 30 dias.

Para Dielman et al.(1991), um método com o qual se tem que lidar no caso dos não envolvidos com a literatura da pesquisa é o da validade do relato do adolescente quanto ao seu próprio uso. A literatura neste campo pode ser sumarizada pelo comentário geral que o relato do próprio adolescente é satisfatório no que diz respeito à confiabilidade e validade, uma vez que o pesquisador garanta aos alunos que suas respostas serão confidenciais (não necessariamente anônimas).

Quanto aos tipos de drogas mais usados pelos 43 estudantes da presente pesquisa, constatou-se que os solventes voláteis ou inalantes vêm em primeiro lugar, cerca de $72,8 \%$. Os que usaram maconha compreendiam $18,2 \% ; 3,6 \%$ já usaram cocaina; $1,8 \%$ fez uso de crack; $1,8 \%$ de "liquid paper" (um dado curioso) e 1,8 utilizou censo (droga não identificada).

Segundo o MINISTÉRIO DA SAÚDE (1991), os estudantes brasileiros apresentaram surpreendente homogeneidade, de Norte a Sul do país, no tocante ao tipo de substância psicoativa preferida. Dados levantados por diferentes cidades e em duas pesquisas mais amplas abrangendo cerca de 47.000 estudantes de 17 cidades do Brasil, em escolas públicas e particulares, apontaram que as drogas mais utilizadas (exceto álcool e tabaco) são os 
solventes voláteis ou inalantes $(17 \%$ dos escolares já os experimentaram de acordo com inquérito realizado em 1989). Nos Estados Unidos, no mesmo ano, o consumo freqüente de maconha e cocaina atingiu as taxas de 6,8\% e 0,9\%, respectivamente, enquanto no Brasil as taxas correspondentes foram $0,5 \%$ e $0,1 \%$, ou seja, o consumo entre os estudantes brasileiros é dez vezes menor, aproximadamente, que entre os estudantes americanos.

Conforme TABELA 4, ao se questionar sobre o motivo que levou os entrevistados a usar drogas, constatou-se que $46,8 \%$ foi por curiosidade, $42,5 \%$ através da influência de amigos, $8,5 \%$ em conseqüência de problemas familiares, $2,2 \%$ outros motivos (curtir o show), o que corresponde a apenas 1 estudante, enquanto nenhum respondeu que foi por causa de problemas sócioeconômicos. Analisando-se as escolas separadamente, verificou-se que os estudantes das escolas públicas, cerca de $51,8 \%$, afirmaram que foi a influência de amigos que os levou a usar drogas; entretanto os estudantes das escolas particulares responderam que o motivo pelo qual usaram drogas foi a curiosidade, o que corresponde a $66,7 \%$ das respostas.

TABELA 4 - DISTRIBUIÇÃO DA FREQÜÊNCIA DOS MOTIVOS QUE LEVARAM OS ESCOLARES DE $7^{\mathrm{a}}$ E $8^{\mathrm{a}}$ SÉRIES, ENTRE 12 E 15 ANOS DE IDADE, A FAZEREM USO DE DROGAS, ALFENAS - MG. 1993.

\begin{tabular}{l|c|c|c|c|c|c}
\hline \multirow{2}{*}{ MOTIVOS } & \multicolumn{6}{|c}{ ESCOLAS } \\
\cline { 2 - 7 } & \multicolumn{2}{|c|}{ PÚELICAS } & \multicolumn{2}{c|}{ PARTICULARES } & \multicolumn{2}{c}{ PUBL.+PARTIC. } \\
\cline { 2 - 7 } & F & $\%$ & F & $\%$ & F & $\%$ \\
\hline CURIOSIDADE & 10 & 34,5 & 12 & 66,7 & 22 & 46,8 \\
INFLUEENCIADE AMIGOS & 15 & 51,8 & 05 & 27,8 & 20 & 42,5 \\
PROBLEMAS FAMILIARES & 03 & 10,3 & 01 & 05,5 & 04 & 08,5 \\
PROB.SÓCIO-ECONOMMICOS & - & - & - & - & - & - \\
OUTROS & 01 & 03,4 & - & - & 01 & 02,2 \\
\hline TOTAL & 29 & 100,0 & 18 & 100,0 & 47 & 100,0 \\
\hline
\end{tabular}

Murad (1982) realizou duas pesquisas sobre o abuso de drogas entre jovens - uma no curso secundário e outra no curso superior - aplicando 2230 questionários, obtendo, como resposta à pergunta sobre os motivos do uso de drogas, a curiosidade em primeiro lugar (42\% no primeiro caso e $52 \%$ no segundo, entre os usuários). Os jovens são, por sua própria natureza, pelo período que atravessam (a adolescência), curiosos. É um período de transição muito difícil, cheio de interrogações, no qual eles tendem a experimentar "tudo" que lhe vem à mão. É o período no qual ele começa a se auto afirmar, através de experiências vividas positivamente ou, muitas vezes, não tão gratificantes.

Murad (1982) verificou que, em mais de $80 \%$ dos casos, os jovens usuários têm problemas familiares graves: pais separados ou agressivos um com outro, ou com os filhos; a falta de diálogo dentro de casa; falta de apoio familiar e/ou compreensão; rivalidade entre irmãos; falta de carinho; ausência de amor; pais 
que trabalham fora de casa o dia inteiro. Um especialista já escreveu certa vez que "alguns jovens buscam nas drogas a mãe ou o pai que nunca tiveram".

Embora o jovem passe por todos esses problemas, estes não constituem o principal motivo que levam os estudantes entrevistados a fazer uso de drogas, como pode ser verificado através da TABELA 4.

"Curiosidade" e "influência dos amigos", de maneira geral, não tem diferença. No entanto, verifica-se que, separadamente, entre as escolas, isso não é constatado, pois entre os estudantes das escolas públicas sobressai o percentual de influência dos amigos e, nas particulares, caracteriza-se a curiosidade.

Para Murad (1985), o adolescente tem grande desejo de se relacionar e de ser aceito por pessoas de sua própria idade. Para um certo número de jovens, uma das razões mais importantes para experimentar drogas é a necessidade de pertencer a um grupo, ou de se impor nele. Se uma turma extremamente popular usa drogas, o adolescente, visando a sua aceitação pelo grupo, será fortemente tentado a fazer o mesmo.

Quando se questionou se os estudantes receberam alguma orientação sobre drogas, segundo a TABELA $5,98,8 \%$ dos entrevistados das escolas públicas responderam que sim e apenas 1,2\% não; entre as escolas particulares, $96,3 \%$ dos estudantes disseram que já haviam recebido orientação sobre drogas e simplesmente $3,7 \%$ nunca receberam orientação.

TABELA 5 - FREQÜÊNCIA DE ALUNOS DE $7^{\mathrm{a}}$ E $8^{\mathrm{a}}$ SÉRIES, NA FAIXA ETÁRIA DE 12 A 15 ANOS, QUE JÁ RECEBERAM ORIENTAÇÃO SOBRE DROGAS, ALFENAS - MG - 1993.

\begin{tabular}{|c|c|c|c|c|c|}
\hline & \multirow{3}{*}{ ORIENTADO } & \multicolumn{4}{|c|}{ ESCOLAS } \\
\hline & & \multicolumn{2}{|c|}{ PÚBLIC:AS } & \multicolumn{2}{|c|}{ PARTICULARES } \\
\hline & & $\mathbf{F}$ & $\%$ & $\mathbf{F}$ & $\%$ \\
\hline SIM & & 553 & 98,8 & 206 & 96,3 \\
\hline NÃO & & 007 & 01,2 & 008 & 03,7 \\
\hline & TOTAL & 560 & 100,0 & 214 & 100,0 \\
\hline
\end{tabular}

realizadas anteriormente constatou-se que as orientações eram esporádicas.

$O$ resultado sobre a fonte de orientação sobre drogas é evidenciado na TABELA 6. 
TABELA 6 - DISTRIBUIÇÃO DAS FONTES DE ORIENTAÇÃO SOBRE DROGAS RECEBIDAS PELOS ALUNOS DE $7^{\text {a }}$ E $8^{a^{3}}$ SÉRIES, COM IDADE ENTRE 12 E 15 ANOS, ALFENAS - MG - 1993.

\begin{tabular}{|c|c|c|c|c|}
\hline \multirow{3}{*}{ FONTE DE INFORMAÇÃO } & \multicolumn{4}{|c|}{ ESCCLAS } \\
\hline & \multicolumn{2}{|c|}{ PÚBLICAS } & \multicolumn{2}{|c|}{ PARTICULARES } \\
\hline & $\mathbf{F}$ & $\%$ & $\mathbf{F}$ & $\%$ \\
\hline FAMILIARES & 352 & 26,81 . & 175 & 38,7 \\
\hline TELEVISÃO & 278 & 21,20 & 128 & 28,3 \\
\hline ESCOLA & 519 & 39,50 & 100 & 22,2 \\
\hline AMIGO & 164 & 12,50 & 049 & 10,8 \\
\hline TOTAL & 1313 & 100,00 & 452 & 100,0 \\
\hline
\end{tabular}

Conforme Wordaki (1990), o comportamento dos pais a respeito de drogas exerce uma influência importante. A familia é a "influência crucial nos valores e comportamento das crianças". Em casa, jovens podem encontrar estrutura e orientação dos entes queridos que realmente se preocupam com eles. Expectativas claras sobre consumo de drogas podem ser comunicadas. Crianças mais jovens são especialmente vulneráveis às pressões e precisam de um lugar confortável e segurança para ajudar no domínio de suas ansiedades e frustrações.

A ORGANIZACIÓN MUNDIAL DE LA SALUD (1993) alerta os pais sobre tudo, em especial tomar tempo para falar com os filhos, especialmente se eles se interessarem pelo tema. "Dê-lhes a confiança de saber que você se preocupa e os ajudará se tiver algum problema. Tudo isto é importante porque os jovens que possuem boas relações com seus pais têm menor probabilidade de querer usar drogas", recomenda a publicação.

Para Zago (1988), as campanhas antidrogas através da mídia podem parecer uma dupla mensagem, já que, ao mesmo tempo, todos sabem do poder que os meios de comunicação de massa, a televisão por exemplo, têm para aliciar as pessoas no que se refere às outras adicções de bebidas, cigarros, medicamentos etc. $O$ mais doloroso é perceber que geralmente o questionamento sobre drogas, através da mídia, sempre tem como pano de fundo outras intenções, como, por exemplo, a campanha política. A informação contra as drogas veiculada pelos meios de comunicação é muito arriscada, uma vez que pode ser confundida com campanha antidrogadicto, o que, em vez de solucionar, agravaria ainda mais o problema.

A escola é uma instituição social que promove, além da instrução, a educação de crianças e jovens. Para Murad (1985), atribuem-se à escola responsabilidades importantes sob o ponto de vista social, como, por exemplo, educação sobre sexo, trânsito, ecologia e agora tem-se-lhe cobrado a solução do problema da droga entre os jovens. Só que, antes que os professores 
possam ensinar, eles necessitam saber muitos fatos a respeito das drogas e dos adolescentes. Aliado a isso, Zago (1988) comenta que, no $1^{\circ}$ e $2^{\circ}$ Graus, a preocupação é apenas com um programa didático meramente informativo. Com uma formação deficiente e pouco motivado no seu trabalho, o professor se sente como num campo minado ao discutir assuntos que colocam em jogo a formação do aluno. Desse modo, evitando falar de drogas, e despreparado para isso, o professor se esquiva de pisar num terreno desconhecido.

A orientação sobre drogas, recebida através de um amigo, ficou em último lugar, talvez porque a maioria dos amigos em vez de orientar, esclarecer, induzem o colega a usar drogas, o que foi evidenciado pela TABELA 6.

É curioso notar que houve uma diferença nas respostas em relação à principal fonte de orientação recebida pelos estudantes. A resposta dos entrevistados das escolas públicas citou a escola como a principal fonte de orientação; por outro lado, nas escolas particulares, os familiares foram tidos como principais orientadores. Seria importante a realização de outros estudos para constatar o porquê desta diferença.

Apesar de todos esses problemas, ainda não se encontrou um substituto da Escola no seu papel de promotora da educação, afirma Vizzolto (1987), e ela é a que possui melhores condições para sistematizar a informação, segundo os diferentes graus de ensino, de interesse e maturidade dos alunos. No currículo ficam asseguradas a ação informativa, a assiduidade, a continuidade, sendo necessário que o professor em suas aulas proceda de maneira democrática, despido de preconceitos, falso moralismo e com uma postura científica. A escola é também a instituição que possui maiores condições de executar um programa de prevenção, pois retém a clientela de maior risco, ou seja, crianças, adolescentes e jovens.

Acredita-se que no contexto de qualquer orientação, e Murad (1985) confirma isso, é importante ensinar, também, aos jovens a gostarem de si mesmos e a se relacionarem construtivamente com outros jovens, a aprenderem a tomar decisões, a resolverem seus problemas, dentre eles, o uso de drogas.

\section{CONCLUSÃO}

O presente trabalho permite as seguintes conclusões:

- a taxa de consumo de drogas entre os estudantes na faixa etária estudada é baixa, $(5,5 \%)$ em comparação com outros estudos. Embora esse percentual não seja expressivo, é alarmante, é preocupante, visto ter-se encontrado entre eles usuários de cocaína e até crack, drogas consideradas pesadas.

- as drogas a que esses estudantes têm mais acesso são os solventes voláteis ou inalantes e, em segundo lugar, a maconha. A motivação para usar as drogas analisadas foi atribuída à influência dos amigos e à curiosidade. 
- apesar de todos os problemas por que passam as escolas e as familias brasileiras, elas continuam sendo os meios mais comuns pelos quais os estudantes recebem orientações sobre drogas.

- conforme vários estudos, a faixa etária dos estudantes que se tornam usuários de drogas é cada vez menor; sendo assim, não existe uma faixa etária ideal para se começar a dar orientações sobre drogas. Esta informação deve ocorrer o quanto antes, o mais cedo possivel; adequando o nivel de conhecimento à maturidade das crianças.

Um dado animador da pesquisa: é a baixa prevalência do uso de drogas entre jovens estudantes (12 a 15 anos de idade), o que permite uma conclusão promissora: este é o momento propício para que órgãos responsáveis pela saúde pública no município comecem a desenvolver um programa de prevenção de drogas entre tal população.

ABSTRACT: The present paper deals with aspects on drug abuse among 12-15 year old students from the seventh and eighth series from public and private schools in Alfenas in 1993. The most offered and used drugs were the volatile solvents or inhalants followed by marijuana. Drugs are more frequently offered at public places (gymnasia, bars, video game houses, amusement parks, etc.). It was found that $5.5 \%$ of the interviewed students had already used drugs under the influence of friends or out of curiosity. Practically, all students had been informed about drugs either at home and/or at school. As school has the greatest clientele under the risk of drug abuse, that is, children, adolescents and youngsters, and as it is responsible for education and culture communication, it is the most capable one to carry out a drug prevention program.

KEYWORDS: Drugs - Students - School. 
ANEXO

PESQUISA SOBRE DROGAS - PET/ENFERMAGEM

Sua resposta é muito importante para realização desta pesquisa VOCÊ NĀO SERÁ IDENTIFICADO

SEXO IDADE: ESTADO CIVIL:

\section{QUESTIONÁRIO}

01 - JÁ LHE OFERECERAM ALGUM TIPO DE DROGA ?

( ) Sim ( ) Não

02 - CASO A RESPOSTA SEJA AFIRMATIVA, CITE QUAL (OU QUAIS)?

03 - MARQUE COM UM X O LOCAL ONDE LHE OFERECERAM A DROGA:

( ) Casa de amigo

( ) Festa

( ) Escola

( ) Lugar público (ginásios de show, bares, casa de jogos, parque etc.)

04 -VOCÉ CONHECE ALGUM COLEGA NA ESCOLA QUE UTILIZA OU UTILIZAVA DROGAS?
( ) Sim
( ) Não

05 - MARQUE COM UM X OS SINTOMAS APRESENTADOS POR PESSOAS QUE UTILIZAM DROGAS:

( ) Boca seca

( ) Riso e choro fácil

( ) Fala enrolada

( ) Tremor

( ) Olhos vermelhos

( ) Nariz escorrendo

( ) Sonolência

( ) Tagarelice

( ) Não prestar atenção a nada

( ) Dificuldade de pensar claramente

( ) Alucinação (auditiva, visual etátil) 
06 - VOCÊ JÁ USOU QUALQUER TIPO DE DROGA ?

( ) $\operatorname{Sim}$ ( ) Não

07 - CASO A RESPOSTA ANTERIOR TENHA SIDO AFIRMATIVA, ESCREVA O(S) NOME(S) DA(S) DROGA(S):

08 - CASO A RESPOSTA No 6 TENHA SIDO AFIRMATIVA, MARQUE COM UM X O(S) MOTIVO(S) QUE O LEVOU A TAL ATITUDE:

( ) Curiosidade ( ) Problemas familiares

( ) Influência dos amigos ( ) Problemas sócio econômicos

09 - VOCÊ JÁ RECEBEU ALGUMA ORIENTAÇÃO SOBRE DROGAS ?

( ) $\operatorname{Sim}$ ( ) Não

10 - CASO AFIRMATIVO, MARQUE COM UM X ONDE VOCÊ OBTEVE ESTA ORIENTAÇÃO.

( ) Familiares ( ) Escola

( ) Televisão ( ) Amigo

OBRIGADO PELAS RESPOSTAS 


\section{REFERÊNCIAS BIBLIOGRÁFICAS}

1. CHARBONNEAU, P. E. A escola e combate as drogas. In: Drogas ameaça ao futuro. Federação do Comércio do Estado de São Paulo, 1987.

2. ChaRbonneAU, P. E. et al. Pais, filhos e tóxicos. São Paulo: Almed, $1983.88 \mathrm{p}$.

3. DIELMAN, T. E. et al. Environmental correlates of adolescent substance use and misuse: implications for prevention programs. The International Journal of the Addictions. Michigan, n. 25, p. 855-880, 1991.

4. EMEDIATO, L. F. Geração abandonada. 6.ed. São Paulo: EMW Editores, 1985.

5. FARIAS, F. L. R. O consumo de drogas entre alunos de $1^{\circ}$ e $2^{\circ}$ graus da rede estadual de ensino de Cuiabá. Cuiabá, out. 1992. (Plano de Pesquisa).

6. GALLO, F. P. A. Drogas, entorpecentes e narcóticos. Secretaria de Polícia Civil, Rio de Janeiro, 1984.

7. MURAD, J. E. Como enfrentar o abuso de drogas. 3.ed. Belo Horizonte, 1985.

8. MURAD, J. E. O problema dos tóxicos na Universidade, In: Drogas e drogados: $o$ individuo, a familia, a sociedade. São Paulo: Pedagógica e Universitária, 1982. p. 203-27.

9. MURAD, J. E. O que você deve saber sobre os psicotrópicos; a viagem sem bilhete de volta. Belo Horizonte, 1972.

10. NA CARTEIRA AO LADO, Revista Veja. São Paulo, p.43, 27 de Março de 1991.

11. ORGANIZACION MUNDIAL DE LA SALUD. Las enfermeras responden al abuzo de drogas. Consejo Internacional de Enfermeras, 1993.

12. SANCHES, A. M. T. SANCHES, V. F. O consumo de maconha no curso de segundo grau - um estudo exploratório. Drogas e drogados; o individuo, a familia, a sociedade. São Paulo: Pedagógica e Universitária, 1982. p. 143-201.

13. VIZZOLTO, S.M. A droga; a escola e a prevenção. Petrópolis: Vozes, p.57-95, 1987.

14. WODARSKI, J. S. Adolescent substance abuse: practice implications. Adolescence, São Diego: Libra Publishers, v.25, n.99, p. 667-88, 1990.

15. ZAGO, J. A. Drogas: condições psicossociais da dependência. São Paulo: Icone, 1988. $100 \mathrm{p}$. 